\title{
Coupled fixed point theorems for generalized contractive mappings in partially ordered $G$-metric spaces
}

\author{
Rabian Wangkeeree ${ }^{1,2^{*}}$ and Thanatporn Bantaojai
}

\section{"Correspondence:}

rabianw@nu.ac.th

'Department of Mathematics,

Faculty of Science, Naresuan

University, Phitsanulok, 65000,

Thailand

${ }^{2}$ Centre of Excellence in

Mathematics, CHE, Si Ayutthaya

Road, Bangkok, 10400, Thailand

\begin{abstract}
In this paper, we establish some coupled coincidence and coupled common fixed point theorems for nonlinear contractive mappings having the mixed monotone property in partially ordered G-metric spaces. The results on fixed point theorems are generalizations of the recent results of Choudhury and Maity (Math. Comput. Model. 54:73-79, 2011) and Luong and Thuan (Math. Comput. Model. 55:1601-1609, 2012).
\end{abstract}

Keywords: G-metric space; ordered set; coupled coincidence point; coupled fixed point; mixed g-monotone property

\section{Introduction and preliminaries}

One of the simplest and the most useful results in the fixed point theory is the BanachCaccioppoli contraction [1] mapping principle, a power tool in analysis. This principle has been generalized in different directions in different spaces by mathematicians over the years (see [2-10] and references mentioned therein). On the other hand, fixed point theory has received much attention in metric spaces endowed with a partial ordering. The first result in this direction was given by Ran and Reurings [11] and they presented applications of their results to matrix equations. Subsequently, Nieto and Rodríguez-López [12] extended the results in [11] for non-decreasing mappings and obtained a unique solution for a first-order ordinary differential equation with periodic boundary conditions (see also [13-19]).

In recent times, fixed point theory has developed rapidly in partially ordered metric spaces, that is, metric spaces endowed with a partial ordering. Some of these works are noted in [13, 17, 20,21]. Bhaskar and Lakshmikantham [21] introduced the concept of a coupled fixed point and the mixed monotone property. Furthermore, they proved some coupled fixed point theorems for mappings which satisfy the mixed monotone property and gave some applications in the existence and uniqueness of a solution for a periodic boundary value problem. After the publication of this work, several coupled fixed point and coincidence point results have appeared in the recent literature. Works noted in [22$25]$ are some examples of these works.

Mustafa and Sims [26, 27] introduced a new structure of generalized metric spaces, which are called $G$-metric spaces, as a generalization of metric spaces to develop and introduce a new fixed point theory for various mappings in this new structure. Later, several fixed point theorems in G-metric spaces were obtained by [28-34].

(c) 2012 Wangkeeree and Bantaojai; licensee Springer. This is an Open Access article distributed under the terms of the Creative Commons Attribution License (http://creativecommons.org/licenses/by/2.0), which permits unrestricted use, distribution, and reproduction in any medium, provided the original work is properly cited. 
To fix the context in which we are placing our results, recall the following notions. Throughout this article, $(X, \preceq)$ denotes a partially ordered set with the partial order $\preceq$. By $x \prec y$, we mean $x \preceq y$ but $x \neq y$. A mapping $g: X \rightarrow X$ is said to be non-decreasing (non-increasing) if for all $x, y \in X, x \preceq y$ implies $g(x) \preceq g(y)(g(y) \preceq g(x)$, respectively).

The concept of a mixed monotone property has been introduced by Bhaskar and Lakshmikantham [21].

Definition 1.1 [21] Let $(X, \preceq)$ be a partial ordered set. A mapping $F: X \times X \rightarrow X$ is said to be have the mixed monotone property if $F(x, y)$ is monotone non-decreasing in $x$ and is monotone non-increasing in $y$, that is, for any $x, y \in X$,

$$
x_{1}, x_{2} \in X, \quad x_{1} \preceq x_{2} \quad \Longrightarrow \quad F\left(x_{1}, y\right) \preceq F\left(x_{2}, y\right)
$$

and

$$
y_{1}, y_{2} \in X, \quad y_{1} \preceq y_{2} \quad \Longrightarrow \quad F\left(x, y_{1}\right) \succeq F\left(x, y_{2}\right) .
$$

The following concepts were introduced in [35].

Definition 1.2 [35] Let $(X, \preceq)$ be a partial ordered set and $F: X \times X \rightarrow X$ and $g: X \rightarrow X$ be two mappings. We say that $F$ has the mixed $g$-monotone property if $F(x, y)$ is $g$-monotone non-decreasing in $x$ and it is $g$-monotone non-increasing in $y$, that is, for any $x, y \in X$

$$
x_{1}, x_{2} \in X, \quad g\left(x_{1}\right) \preceq g\left(x_{2}\right) \quad \Longrightarrow \quad F\left(x_{1}, y\right) \preceq F\left(x_{2}, y\right)
$$

and

$$
y_{1}, y_{2} \in X, \quad g\left(y_{1}\right) \preceq g\left(y_{2}\right) \quad \Longrightarrow \quad F\left(x, y_{1}\right) \succeq F\left(x, y_{2}\right) .
$$

Definition 1.3 [21,35] Let $F: X \times X \rightarrow X$ and $g: X \rightarrow X$ be mappings. An element $(x, y) \in$ $X \times X$ is said to be:

(i) a coupled fixed point of a mapping $F$ if

$$
x=F(x, y) \quad \text { and } \quad y=F(y, x) \text {; }
$$

(ii) a coupled coincidence point of mapping $F$ and $g$ if

$$
g(x)=F(x, y) \quad \text { and } \quad g(y)=F(y, x)
$$

(iii) a coupled common fixed point of mappings $F$ and $g$ if

$$
x=g(x)=F(x, y) \quad \text { and } \quad y=g(y)=F(y, x) .
$$

Consistent with Mustafa and Sims [26, 27], the following definitions and results will be needed in the sequel. 
Definition 1.4 (G-metric space [27]) Let $X$ be a non-empty set. Let $G: X \times X \times X \rightarrow \mathbb{R}_{+}$ be a function satisfying the following properties:

(G1) $G(x, y, z)=0$ if $x=y=z$;

(G2) $G(x, x, y)>0$ for all $x, y \in X$ with $x \neq y$;

(G3) $G(x, x, y) \leq G(x, y, z)$ for all $x, y, z \in X$ with $z \neq y$;

(G4) $G(x, y, z)=G(x, z, y)=G(y, z, x)=\cdots$ (symmetry in all three variables);

(G5) $G(x, y, z) \leq G(x, a, a)+G(a, y, z)$ for all $x, y, z, a \in X$ (rectangle inequality).

Then the function $G$ is called a $G$-metric on $X$ and the pair $(X, G)$ is called a $G$-metric space.

Definition 1.5 [27] Let $X$ be a $G$-metric space, and let $\left\{x_{n}\right\}$ be a sequence of points of $X$, a point $x \in X$ is said to be the limit of a sequence $\left\{x_{n}\right\}$ if $G\left(x, x_{n}, x_{m}\right) \rightarrow 0$ as $n, m \rightarrow \infty$ and sequence $\left\{x_{n}\right\}$ is said to be $G$-convergent to $x$.

From this definition, we obtain that if $x_{n} \rightarrow x$ in a $G$-metric space $X$, then for any $\varepsilon>0$, there exists a positive integer $N$ such that $G\left(x, x_{n}, x_{m}\right)<\varepsilon$ for all $n, m \geq N$.

It has been shown in [27] that the G-metric induces a Hausdorff topology and the convergence described in the above definition is relative to this topology. So, a sequence can converge, at the most, to one point.

Definition 1.6 [27] Let $X$ be a $G$-metric space, a sequence $\left\{x_{n}\right\}$ is called G-Cauchy if for every $\varepsilon>0$, there is a positive integer $N$ such that $G\left(x_{n}, x_{m}, x_{l}\right)<\varepsilon$ for all $n, m, l \geq N$, that is, if $G\left(x_{n}, x_{m}, x_{l}\right) \rightarrow 0$, as $n, m, l \rightarrow \infty$.

We next state the following lemmas.

Lemma 1.7 [27] If $X$ is a G-metric space, then the following are equivalent:

(1) $\left\{x_{n}\right\}$ is G-convergent to $x$.

(2) $G\left(x_{n}, x_{n}, x\right) \rightarrow 0$ as $n \rightarrow \infty$.

(3) $G\left(x_{n}, x, x\right) \rightarrow 0$ as $n \rightarrow \infty$.

(4) $G\left(x_{m}, x_{n}, x\right) \rightarrow 0$ as $n, m \rightarrow \infty$.

Lemma 1.8 [27] If $X$ is a G-metric space, then the following are equivalent:

(1) the sequence $\left\{x_{n}\right\}$ is G-Cauchy;

(2) for every $\varepsilon>0$, there exists a positive integer $N$ such that $G\left(x_{n}, x_{m}, x_{m}\right)<\varepsilon$, for all $n, m \geq N$.

Lemma 1.9 [27] If $X$ is a G-metric space, then $G(x, y, y) \leq 2 G(y, x, x)$ for all $x, y \in X$.

Lemma 1.10 If $X$ is a G-metric space, then $G(x, x, y) \leq G(x, x, z)+G(z, z, y)$ for all $x, y, z \in X$.

Definition 1.11 [27] Let $(X, G),\left(X^{\prime}, G^{\prime}\right)$ be two generalized metric spaces. A mapping $f$ : $X \rightarrow X^{\prime}$ is $G$-continuous at a point $x \in X$ if and only if it is $G$ sequentially continuous at $x$, that is, whenever $\left\{x_{n}\right\}$ is $G$-convergent to $x,\left\{f\left(x_{n}\right)\right\}$ is $G^{\prime}$-convergent to $f(x)$.

Definition 1.12 [27] A G-metric space $X$ is called a symmetric $G$-metric space if

$$
G(x, y, y)=G(y, x, x)
$$

for all $x, y \in X$. 
Definition 1.13 [27] A G-metric space $X$ is said to be $G$-complete (or a complete G-metric space) if every $G$-Cauchy sequence in $X$ is convergent in $X$.

Definition 1.14 Let $X$ be a $G$-metric space. A mapping $F: X \times X \rightarrow X$ is said to be continuous if for any two $G$-convergent sequences $\left\{x_{n}\right\}$ and $\left\{y_{n}\right\}$ converging to $x$ and $y$, respectively, $\left\{F\left(x_{n}, y_{n}\right)\right\}$ is $G$-convergent to $F(x, y)$.

Definition 1.15 Let $X$ be a non-empty set and $F: X \times X \rightarrow X$ and $g: X \rightarrow X$ two mappings. We say $F$ and $g$ are commutative (or that $F$ and $g$ commute) if

$$
g(F(x, y))=F(g(x), g(y)), \quad \forall x, y \in X
$$

Recently, Choudhury and Maity [36] studied necessary conditions for the existence of a coupled fixed point in partially ordered G-metric spaces. They obtained the following interesting result.

Theorem 1.16 [36] Let $(X, \preceq)$ be a partially ordered set such that $X$ is a complete G-metric space and $F: X \times X \rightarrow X$ be a mapping having the mixed monotone property on $X$. Suppose there exists $k \in[0,1)$ such that

$$
G(F(x, y), F(u, v), F(w, z)) \leq \frac{k}{2}(G(x, u, w)+G(y, v, z))
$$

for all $x, y, z, u, v, w \in X$ for which $x \succeq u \succeq w$ and $y \preceq v \preceq z$, where either $u \neq w$ or $v \neq z$. If there exists $x_{0}, y_{0} \in X$ such that

$$
x_{0} \preceq F\left(x_{0}, y_{0}\right) \quad \text { and } \quad y_{0} \succeq F\left(y_{0}, x_{0}\right)
$$

and either

(a) $F$ is continuous or

(b) $X$ has the following property:

(i) if a non-decreasing sequence $\left\{x_{n}\right\}$ is such that $x_{n} \rightarrow x$, then $x_{n} \preceq x$ for all $n$,

(ii) if a non-decreasing sequence $\left\{y_{n}\right\}$ is such that $y_{n} \rightarrow y$, then $y \leq y_{n}$ for all $n$,

then $F$ has a coupled fixed point.

Let $\Theta$ denote the class of all functions $\varphi:[0, \infty) \times[0, \infty) \rightarrow[0, \infty)$ satisfying the following condition:

$$
\lim _{t_{1} \rightarrow r_{1}, t_{2} \rightarrow r_{2}} \varphi\left(t_{1}, t_{2}\right)>0
$$

for all $\left(r_{1}, r_{2}\right) \in[0, \infty) \times[0, \infty)$ with $r_{1}+r_{2}>0$.

Remark 1.17 If the function $\varphi:[0, \infty) \times[0, \infty) \rightarrow[0, \infty)$ satisfies (1.4), then, for any $t_{1}, t_{2} \in[0, \infty)$ with either $t_{1} \neq 0$ or $t_{2} \neq 0, \varphi\left(t_{1}, t_{2}\right)>0$. Indeed, suppose that $t_{1} \neq 0$, we have $t_{1}+t_{2}>0$. Taking $t_{n}^{1}=t_{1}$ and $t_{n}^{2}=t_{2}$ for all $n \in \mathbb{N}$, we have, by (1.4), that

$$
\varphi\left(t_{1}, t_{2}\right)=\lim _{t_{n}^{1} \rightarrow t_{1}, t_{n}^{2} \rightarrow t_{2}} \varphi\left(t_{n}^{1}, t_{n}^{2}\right)>0 .
$$


Example 1.18 The following are some examples of $\varphi$, for all $\left(t_{1}, t_{2}\right) \in[0, \infty) \times[0, \infty)$,

(1) $\varphi\left(t_{1}, t_{2}\right)=k \max \left\{t_{1}, t_{2}\right\}$ for $k>0$;

(2) $\varphi\left(t_{1}, t_{2}\right)=a t_{1}^{p}+b t_{2}^{q}$ for $a, b, p, q>0$;

(3) $\varphi\left(t_{1}, t_{2}\right)=\frac{1-k}{2}\left(t_{1}+t_{2}\right)$ for some $k \in[0,1)$.

Using basically these concepts, Luong and Thuan [37] proved the following coupled fixed point theorem for nonlinear contractive mappings having the mixed monotone property in partially ordered $G$-metric spaces.

Theorem 1.19 [37, Theorem 2.1] Let $(X, \leq)$ be a partially ordered set and suppose that there exists a G-metric $G$ on $X$ such that $(X, G)$ is a complete G-metric space. Let $F: X \times$ $X \rightarrow X$ be a mapping having the mixed monotone property on $X$. Suppose that there exists $\varphi \in \Theta$ such that

$$
G(F(x, y), F(u, v), F(w, z)) \leq \frac{G(x, u, w)+G(y, v, z)}{2}-\varphi(G(x, u, w), G(y, v, z))
$$

for all $x \succeq u \succeq w$ and $y \preceq v \preceq z$. Suppose that either

(a) $F$ is continuous or

(b) $X$ has the following property:

(i) if a non-decreasing sequence $\left\{x_{n}\right\}$ is such that $x_{n} \rightarrow x$, then $x_{n} \preceq x$ for all $n$,

(ii) if a non-decreasing sequence $\left\{y_{n}\right\}$ is such that $y_{n} \rightarrow y$, then $y \preceq y_{n}$ for all $n$.

If there exist $x_{0}, y_{0} \in X$ such that $x_{0} \preceq F\left(x_{0}, y_{0}\right)$ and $y_{0} \succeq F\left(y_{0}, x_{0}\right)$, then $F$ has a coupled fixed point in $X$.

Starting from the results in Choudhury and Maity [36] and Luong and Thuan [37], our main aim in this paper is to obtain more general coincidence point theorems and coupled common fixed point theorems for mixed monotone operators $F: X \times X \rightarrow X$ satisfying a contractive condition which is significantly more general that the corresponding conditions (1.3) and (1.5) in [36] and [37], respectively, thus extending many other related results in literature. We also provide an illustrative example in support of our results.

\section{Coupled coincidence points}

The first main result in this paper is the following coincidence point theorem which generalizes [36, Theorem 3.1] and [37, Theorem 2.1].

Theorem 2.1 Let $(X, \preceq)$ be a partially ordered set and $G$ be a $G$-metric on $X$ such that $(X, G)$ is a complete G-metric space. Let $g: X \rightarrow X$ be a mapping and $F: X \times X \rightarrow X$ be a mapping having the mixed $g$-monotone property on $X$. Suppose that there exists $\varphi \in \Theta$ such that

$$
\begin{aligned}
M_{F}^{G}(x, u, w, y, v, z) \leq & {[G(g(x), g(u), g(w))+G(g(y), g(v), g(z))] } \\
& -2 \varphi(G(g(x), g(u), g(w)), G(g(y), g(v), g(z)))
\end{aligned}
$$

for all $x, y, z, u, v, w \in X$ for which $g(x) \succeq g(u) \succeq g(w)$ and $g(y) \preceq g(v) \preceq g(z)$ where

$$
M_{F}^{G}(x, u, w, y, v, z)=G(F(x, y), F(u, v), F(w, z))+G(F(y, x), F(v, u), F(z, w)) .
$$


If there exists $x_{0}, y_{0} \in X$ such that

$$
g\left(x_{0}\right) \preceq F\left(x_{0}, y_{0}\right) \quad \text { and } \quad g\left(y_{0}\right) \succeq F\left(y_{0}, x_{0}\right) \text {, }
$$

and suppose $F: X \times X \subseteq g(X), g$ is continuous and commutes with $F$, and also suppose either

(a) $F$ is continuous or

(b) $X$ has the following property:

(i) if a non-decreasing sequence $\left\{x_{n}\right\}$ is such that $x_{n} \rightarrow x$, then $x_{n} \preceq x$ for all $n$,

(ii) if a non-decreasing sequence $\left\{y_{n}\right\}$ is such that $y_{n} \rightarrow y$, then $y \preceq y_{n}$ for all $n$,

then $F$ and $g$ have a coupled coincidence point, that is, there exists $(x, y) \in X \times X$ such that $g(x)=F(x, y)$ and $g(y)=F(y, x)$.

Proof Let $x_{0}, y_{0} \in X$ such that $g\left(x_{0}\right) \preceq F\left(x_{0}, y_{0}\right)$ and $F\left(y_{0}, x_{0}\right) \preceq g\left(y_{0}\right)$. Since $F(X \times X) \subseteq$ $g(X)$, we can choose $x_{1}, y_{1} \in X$ such that $g\left(x_{1}\right)=F\left(x_{0}, y_{0}\right)$ and $g\left(y_{1}\right)=F\left(y_{0}, x_{0}\right)$. Again since $F(X \times X) \subseteq g(X)$, we can choose $x_{2}, y_{2} \in X$ such that $g\left(x_{2}\right)=F\left(x_{1}, y_{1}\right)$ and $g\left(y_{2}\right)=F\left(y_{1}, x_{1}\right)$. Continuing this process, we can construct sequences $\left\{x_{n}\right\}$ and $\left\{y_{n}\right\}$ in $X$ such that

$$
g\left(x_{n+1}\right)=F\left(x_{n}, y_{n}\right) \quad \text { and } \quad g\left(y_{n+1}\right)=F\left(y_{n}, x_{n}\right), \quad \text { for all } n \geq 0
$$

Next, we show that

$$
g\left(x_{n}\right) \preceq g\left(x_{n+1}\right) \text { and } g\left(y_{n}\right) \succeq g\left(y_{n+1}\right) \quad \text { for all } n \geq 0 \text {. }
$$

Since $g\left(x_{0}\right) \preceq F\left(x_{0}, y_{0}\right)=g\left(x_{1}\right)$ and $g\left(y_{0}\right) \succeq F\left(y_{0}, x_{0}\right)=g\left(y_{1}\right)$, therefore, (2.3) holds for $n=0$. Next, suppose that (2.3) holds for some fixed $n \geq 0$, that is,

$$
g\left(x_{n}\right) \preceq g\left(x_{n+1}\right) \text { and } g\left(y_{n}\right) \succeq g\left(y_{n+1}\right) .
$$

Since $F$ has the mixed $g$-monotone property, from (2.4) and (1.1), we have

$$
F\left(x_{n}, y\right) \preceq F\left(x_{n+1}, y\right) \quad \text { and } \quad F\left(y_{n+1}, x\right) \preceq F\left(y_{n}, x\right)
$$

for all $x, y \in X$, and from (2.4) and (1.2), we have

$$
F\left(y, x_{n}\right) \succeq F\left(y, x_{n+1}\right) \text { and } F\left(x, y_{n+1}\right) \succeq F\left(x, y_{n}\right) \text {, }
$$

for all $x, y \in X$. If we take $y=y_{n}$ and $x=x_{n}$ in (2.5), then we obtain

$$
g\left(x_{n+1}\right)=F\left(x_{n}, y_{n}\right) \preceq F\left(x_{n+1}, y_{n}\right) \quad \text { and } \quad F\left(y_{n+1}, x_{n}\right) \preceq F\left(y_{n}, x_{n}\right)=g\left(y_{n+1}\right) \text {. }
$$

If we take $y=y_{n+1}$ and $x=x_{n+1}$ in (2.6), then

$$
F\left(y_{n+1}, x_{n}\right) \succeq F\left(y_{n+1}, x_{n+1}\right)=g\left(y_{n+2}\right) \quad \text { and } \quad g\left(x_{n+2}\right)=F\left(x_{n+1}, y_{n+1}\right) \succeq F\left(x_{n+1}, y_{n}\right) \text {. }
$$

Now, from (2.7) and (2.8), we have

$$
g\left(x_{n+1}\right) \preceq g\left(x_{n+2}\right) \quad \text { and } \quad g\left(y_{n+1}\right) \succeq g\left(y_{n+2}\right) \text {. }
$$


Therefore, by the mathematical induction, we conclude that (2.3) holds for all $n \geq 0$. Since $g\left(x_{n}\right) \preceq g\left(x_{n+1}\right)$ and $g\left(y_{n}\right) \succeq g\left(y_{n+1}\right)$ for all $n \geq 0$ so from (2.1), we have

$$
\begin{aligned}
G( & \left.g\left(x_{n+1}\right), g\left(x_{n+1}\right), g\left(x_{n}\right)\right)+G\left(g\left(y_{n+1}\right), g\left(y_{n+1}\right), g\left(y_{n}\right)\right) \\
= & G\left(F\left(x_{n}, y_{n}\right), F\left(x_{n}, y_{n}\right), F\left(x_{n-1}, y_{n-1}\right)\right) \\
& +G\left(F\left(y_{n}, x_{n}\right), F\left(y_{n}, x_{n}\right), F\left(y_{n-1}, x_{n-1}\right)\right) \\
= & M_{F}^{G}\left(x_{n}, x_{n}, x_{n-1}, y_{n}, y_{n}, y_{n-1}\right) \\
\leq & {\left[G\left(g\left(x_{n}\right), g\left(x_{n}\right), g\left(x_{n-1}\right)\right)+G\left(g\left(y_{n}\right), g\left(y_{n}\right), g\left(y_{n-1}\right)\right)\right] } \\
& -2 \varphi\left(G\left(g\left(x_{n}\right), g\left(x_{n}\right), g\left(x_{n-1}\right)\right), G\left(g\left(y_{n}\right), g\left(y_{n}\right), g\left(y_{n-1}\right)\right)\right) .
\end{aligned}
$$

Setting

$$
\omega_{n+1}^{x}:=G\left(g\left(x_{n+1}\right), g\left(x_{n+1}\right), g\left(x_{n}\right)\right)
$$

and

$$
\omega_{n+1}^{y}:=G\left(g\left(y_{n+1}\right), g\left(y_{n+1}\right), g\left(y_{n}\right)\right) \text { for all } n \geq 0,
$$

we have, by (2.10), that

$$
\omega_{n+1}^{x}+\omega_{n+1}^{y} \leq \omega_{n}^{x}+\omega_{n}^{y}-2 \varphi\left(\omega_{n}^{x}, \omega_{n}^{y}\right) .
$$

As $\varphi\left(t_{1}, t_{2}\right) \geq 0$ for all $\left(t_{1}, t_{2}\right) \in[0, \infty) \times[0, \infty)$, we have

$$
\omega_{n+1}^{x}+\omega_{n+1}^{y} \leq \omega_{n}^{x}+\omega_{n}^{y}, \quad \text { for all } n \geq 0
$$

Then the sequence $\left\{\omega_{n}^{x}+\omega_{n}^{y}\right\}$ is decreasing. Therefore, there exists $\omega \geq 0$ such that

$$
\begin{aligned}
\lim _{n \rightarrow \infty}\left(\omega_{n}^{x}+\omega_{n}^{y}\right) & =\lim _{n \rightarrow \infty}\left(G\left(g\left(x_{n+1}\right), g\left(x_{n+1}\right), g\left(x_{n}\right)\right)+G\left(g\left(y_{n+1}\right), g\left(y_{n+1}\right), g\left(y_{n}\right)\right)\right) \\
& =\omega .
\end{aligned}
$$

Now, we show that $\omega=0$. Suppose, to contrary, that $\omega>0$. From (2.12), the sequences $\left\{G\left(g\left(x_{n+1}\right), g\left(x_{n+1}\right), g\left(x_{n}\right)\right)\right\}$ and $\left\{G\left(g\left(y_{n+1}\right), g\left(y_{n+1}\right), g\left(y_{n}\right)\right)\right\}$ have convergent subsequences $\left\{G\left(g\left(x_{n(j)+1}\right), g\left(x_{n(j)+1}\right), g\left(x_{n(j)}\right)\right)\right\}$ and $\left\{G\left(g\left(y_{n(j)+1}\right), g\left(y_{n(j)+1}\right), g\left(y_{n(j)}\right)\right)\right\}$, respectively. Assume that

$$
\lim _{j \rightarrow \infty} \omega_{n(j)+1}^{x}=\lim _{j \rightarrow \infty} G\left(g\left(x_{n(j)+1}\right), g\left(x_{n(j)+1}\right), g\left(x_{n(j)}\right)\right)=\omega_{1}
$$

and

$$
\lim _{j \rightarrow \infty} \omega_{n(j)+1}^{y}=\lim _{j \rightarrow \infty} G\left(g\left(y_{n(j)+1}\right), g\left(y_{n(j)+1}\right), g\left(y_{n(j)}\right)\right)=\omega_{2},
$$

which gives that $\omega_{1}+\omega_{2}=\omega$. From (2.11), we have

$$
\omega_{n(j)+1}^{x}+\omega_{n(j)+1}^{y} \leq \omega_{n(j)}^{x}+\omega_{n(j)}^{y}-2 \varphi\left(\omega_{n(j)}^{x}, \omega_{n(j)}^{y}\right) .
$$


Then taking the limit as $j \rightarrow \infty$ in the above inequality, we obtain

$$
\omega \leq \omega-2 \lim _{j \rightarrow \infty} \varphi\left(\omega_{n(j)}^{x}, \omega_{n(j)}^{y}\right)<\omega,
$$

which is a contradiction. Thus $\omega=0$; that is,

$$
\begin{aligned}
& \lim _{n \rightarrow \infty}\left(G\left(g\left(x_{n+1}\right), g\left(x_{n+1}\right), g\left(x_{n}\right)\right)+G\left(g\left(y_{n+1}\right), g\left(y_{n+1}\right), g\left(y_{n}\right)\right)\right) \\
& \quad=\lim _{n \rightarrow \infty}\left(\omega_{n}^{x}+\omega_{n}^{y}\right)=0 .
\end{aligned}
$$

Next, we show that $\left\{g\left(x_{n}\right)\right\}$ and $\left\{g\left(y_{n}\right)\right\}$ are G-Cauchy sequences. On the contrary, assume that at least one of $\left\{g\left(x_{n}\right)\right\}$ or $\left\{g\left(y_{n}\right)\right\}$ is not a G-Cauchy sequence. By Lemma 1.8, there is an $\varepsilon>0$ for which we can find subsequences $\left\{g\left(x_{n(k)}\right)\right\},\left\{g\left(x_{m(k)}\right)\right\}$ of $\left\{g\left(x_{n}\right)\right\}$ and $\left\{g\left(y_{n(k)}\right)\right\}$, $\left\{g\left(y_{m(k)}\right)\right\}$ of $\left\{g\left(y_{n}\right)\right\}$ with $n(k)>m(k) \geq k$ such that

$$
G\left(g\left(x_{n(k)}\right), g\left(x_{n(k)}\right), g\left(x_{m(k)}\right)\right)+G\left(g\left(y_{n(k)}\right), g\left(y_{n(k)}\right), g\left(y_{m(k)}\right)\right) \geq \varepsilon .
$$

Further, corresponding to $m(k)$, we can choose $n(k)$ in such a way that it is the smallest integer with $n(k)>m(k) \geq k$ and satisfies (2.16). Then

$$
G\left(g\left(x_{n(k)-1}\right), g\left(x_{n(k)-1}\right), g\left(x_{m(k)}\right)\right)+G\left(g\left(y_{n(k)-1}\right), g\left(y_{n(k)-1}\right), g\left(y_{m(k)}\right)\right)<\varepsilon .
$$

By Lemma 1.10, we have

$$
\begin{aligned}
G\left(g\left(x_{n(k)}\right), g\left(x_{n(k)}\right), g\left(x_{m(k)}\right)\right) \leq & G\left(g\left(x_{n(k)}\right), g\left(x_{n(k)}\right), g\left(x_{n(k)-1}\right)\right) \\
& +G\left(g\left(x_{n(k)-1}\right), g\left(x_{n(k)-1}\right), g\left(x_{m(k)}\right)\right)
\end{aligned}
$$

and

$$
\begin{aligned}
G\left(g\left(y_{n(k)}\right), g\left(y_{n(k)}\right), g\left(y_{m(k)}\right)\right) \leq & G\left(g\left(y_{n(k)}\right), g\left(y_{n(k)}\right), g\left(y_{n(k)-1}\right)\right) \\
& +G\left(g\left(y_{n(k)-1}\right), g\left(y_{n(k)-1}\right), g\left(y_{m(k)}\right)\right) .
\end{aligned}
$$

In view of (2.16)-(2.19), we have

$$
\begin{aligned}
\varepsilon \leq & G\left(g\left(x_{n(k)}\right), g\left(x_{n(k)}\right), g\left(x_{m(k)}\right)\right)+G\left(g\left(y_{n(k)}\right), g\left(y_{n(k)}\right), g\left(y_{m(k)}\right)\right) \\
\leq & G\left(g\left(x_{n(k)}\right), g\left(x_{n(k)}\right), g\left(x_{n(k)-1}\right)\right)+G\left(g\left(y_{n(k)}\right), g\left(y_{n(k)}\right), g\left(y_{n(k)-1}\right)\right) \\
& +G\left(g\left(x_{n(k)-1}\right), g\left(x_{n(k)-1}\right), g\left(x_{m(k)}\right)\right)+G\left(g\left(y_{n(k)-1}\right), g\left(y_{n(k)-1}\right), g\left(y_{m(k)}\right)\right) \\
< & G\left(g\left(x_{n(k)}\right), g\left(x_{n(k)}\right), g\left(x_{n(k)-1}\right)\right)+G\left(g\left(y_{n(k)}\right), g\left(y_{n(k)}\right), g\left(y_{n(k)-1}\right)\right)+\varepsilon .
\end{aligned}
$$

Then letting $k \rightarrow \infty$ in the last inequality and using (2.15), we have

$$
\lim _{k \rightarrow \infty}\left[G\left(g\left(x_{n(k)}\right), g\left(x_{n(k)}\right), g\left(x_{m(k)}\right)\right)+G\left(g\left(y_{n(k)}\right), g\left(y_{n(k)}\right), g\left(y_{m(k)}\right)\right)\right]=\varepsilon .
$$

By Lemma 1.9 and Lemma 1.10, we have

$$
\begin{aligned}
G\left(g\left(x_{n(k)}\right), g\left(x_{n(k)}\right), g\left(x_{m(k)}\right)\right) \leq & G\left(g\left(x_{n(k)}\right), g\left(x_{n(k)}\right), g\left(x_{n(k)+1}\right)\right) \\
& +G\left(g\left(x_{n(k)+1}\right), g\left(x_{n(k)+1}\right), g\left(x_{m(k)}\right)\right)
\end{aligned}
$$




$$
\begin{aligned}
\leq & 2 G\left(g\left(x_{n(k)+1}\right), g\left(x_{n(k)+1}\right), g\left(x_{n(k)}\right)\right) \\
& +G\left(g\left(x_{n(k)+1}\right), g\left(x_{n(k)+1}\right), g\left(x_{m(k)+1}\right)\right) \\
& +G\left(g\left(x_{m(k)+1}\right), g\left(x_{m(k)+1}\right), g\left(x_{m(k)}\right)\right)
\end{aligned}
$$

and

$$
\begin{aligned}
G\left(g\left(y_{n(k)}\right), g\left(y_{n(k)}\right), g\left(y_{m(k)}\right)\right) \leq & 2 G\left(g\left(y_{n(k)+1}\right), g\left(y_{n(k)+1}\right), g\left(y_{n(k)}\right)\right) \\
& +G\left(g\left(y_{n(k)+1}\right), g\left(y_{n(k)+1}\right), g\left(y_{m(k)+1}\right)\right) \\
& +G\left(g\left(x_{m(k)+1}\right), g\left(x_{m(k)+1}\right), g\left(x_{m(k)}\right)\right) .
\end{aligned}
$$

It follows from (2.21) and (2.22) that

$$
\begin{aligned}
& G(\left.g\left(x_{n(k)}\right), g\left(x_{n(k)}\right), g\left(x_{m(k)}\right)\right)+G\left(g\left(y_{n(k)}\right), g\left(y_{n(k)}\right), g\left(y_{m(k)}\right)\right) \\
& \leq 2\left(\left\{\omega_{n(k)+1}^{x}+\omega_{n(k)+1}^{y}\right\}\right)+\left(\left\{\omega_{m(k)+1}^{x}+\omega_{m(k)+1}^{y}\right\}\right) \\
& \quad+G\left(g\left(x_{n(k)+1}\right), g\left(x_{n(k)+1}\right), g\left(x_{m(k)+1}\right)\right) \\
& \quad+G\left(g\left(y_{n(k)+1}\right), g\left(y_{n(k)+1}\right), g\left(y_{m(k)+1}\right)\right) .
\end{aligned}
$$

Since $n(k)>m(k)$, we get

$$
g\left(x_{n(k)}\right) \succeq g\left(x_{m(k)}\right) \quad \text { and } \quad g\left(y_{n(k)}\right) \preceq g\left(y_{m(k)}\right),
$$

and also, from (2.1),

$$
\begin{aligned}
G( & \left.g\left(x_{n(k)+1}\right), g\left(x_{n(k)+1}\right), g\left(x_{m(k)+1}\right)\right)+G\left(g\left(y_{n(k)+1}\right), g\left(y_{n(k)+1}\right), g\left(y_{m(k)+1}\right)\right) \\
= & G\left(F\left(x_{n(k)}, y_{n(k)}\right), F\left(x_{n(k)}, y_{n(k)}\right), F\left(x_{m(k)}, y_{m(k)}\right)\right) \\
& +G\left(F\left(y_{n(k)}, x_{n(k)}\right), F\left(y_{n(k)}, x_{n(k)}\right), F\left(y_{m(k)}, x_{m(k)}\right)\right) \\
= & M_{F}^{G}\left(x_{n(k)}, x_{n(k)}, x_{m(k)}, y_{n(k)}, y_{n(k)}, y_{m(k)}\right) \\
\leq & {\left[G\left(g\left(x_{n(k)}\right), g\left(x_{n(k)}\right), g\left(x_{m(k)}\right)\right)+G\left(g\left(y_{n(k)}\right), g\left(y_{n(k)}\right), g\left(y_{m(k)}\right)\right)\right] } \\
& -2 \varphi\left(G\left(g\left(x_{n(k)}\right), g\left(x_{n(k)}\right), g\left(x_{m(k)}\right)\right), G\left(g\left(y_{n(k)}\right), g\left(y_{n(k)}\right), g\left(y_{m(k)}\right)\right)\right) .
\end{aligned}
$$

From (2.23) and (2.24), we have

$$
\begin{aligned}
& 2\left(\left\{\omega_{n(k)+1}^{x}+\omega_{n(k)+1}^{y}\right\}\right)+\left(\left\{\omega_{m(k)+1}^{x}+\omega_{m(k)+1}^{y}\right\}\right) \\
& \geq G\left(g\left(x_{n(k)}\right), g\left(x_{n(k)}\right), g\left(x_{m(k)}\right)\right)+G\left(g\left(y_{n(k)}\right), g\left(y_{n(k)}\right), g\left(y_{m(k)}\right)\right) \\
&-G\left(g\left(x_{n(k)+1}\right), g\left(x_{n(k)+1}\right), g\left(x_{m(k)+1}\right)\right)-G\left(g\left(y_{n(k)+1}\right), g\left(y_{n(k)+1}\right), g\left(y_{m(k)+1}\right)\right) \\
& \geq G\left(g\left(x_{n(k)}\right), g\left(x_{n(k)}\right), g\left(x_{m(k)}\right)\right)+G\left(g\left(y_{n(k)}\right), g\left(y_{n(k)}\right), g\left(y_{m(k)}\right)\right) \\
&-\left[G\left(g\left(x_{n(k)}\right), g\left(x_{n(k)}\right), g\left(x_{m(k)}\right)\right)+G\left(g\left(y_{n(k)}\right), g\left(y_{n(k)}\right), g\left(y_{m(k)}\right)\right)\right] \\
&+2 \varphi\left(G\left(g\left(x_{n(k)}\right), g\left(x_{n(k)}\right), g\left(x_{m(k)}\right)\right), G\left(g\left(y_{n(k)}\right), g\left(y_{n(k)}\right), g\left(y_{m(k)}\right)\right)\right) \\
&= 2 \varphi\left(G\left(g\left(x_{n(k)}\right), g\left(x_{n(k)}\right), g\left(x_{m(k)}\right)\right), G\left(g\left(y_{n(k)}\right), g\left(y_{n(k)}\right), g\left(y_{m(k)}\right)\right)\right) .
\end{aligned}
$$


This implies that

$$
\begin{aligned}
& 2\left(\left\{\omega_{n(k)+1}^{x}+\omega_{n(k)+1}^{y}\right\}\right)+\left(\left\{\omega_{m(k)+1}^{x}+\omega_{m(k)+1}^{y}\right\}\right) \\
& \quad \geq 2 \varphi\left(G\left(g\left(x_{n(k)}\right), g\left(x_{n(k)}\right), g\left(x_{m(k)}\right)\right), G\left(g\left(y_{n(k)}\right), g\left(y_{n(k)}\right), g\left(y_{m(k)}\right)\right)\right) .
\end{aligned}
$$

From (2.20), the sequences $\left\{G\left(g\left(x_{n(k)}\right), g\left(x_{n(k)}\right), g\left(x_{m(k)}\right)\right)\right\}$ and $\left\{G\left(g\left(y_{n(k)}\right), g\left(y_{n(k)}\right), g\left(y_{m(k)}\right)\right)\right\}$ have subsequences converging to, say, $\varepsilon_{1}$ and $\varepsilon_{2}$, respectively, and $\varepsilon_{1}+\varepsilon_{2}=\varepsilon>0$. By passing to subsequences, we may assume that

$$
\lim _{k \rightarrow \infty} G\left(g\left(x_{n(k)}\right), g\left(x_{n(k)}\right), g\left(x_{m(k)}\right)\right)=\varepsilon_{1} \quad \text { and } \quad \lim _{k \rightarrow \infty} G\left(g\left(y_{n(k)}\right), g\left(y_{n(k)}\right), g\left(y_{m(k)}\right)\right)=\varepsilon_{2} \text {. }
$$

Taking $k \rightarrow \infty$ in $(2.25)$ and using (2.15), we have

$$
\begin{aligned}
0= & \lim _{k \rightarrow \infty}\left[2\left(\left\{\omega_{n(k)+1}^{x}+\omega_{n(k)+1}^{y}\right\}\right)+\left(\left\{\omega_{m(k)}^{x}+\omega_{m(k)}^{y}\right\}\right)\right] \\
\geq & \lim _{k \rightarrow \infty} 2 \varphi\left(G\left(g\left(x_{n(k)}\right), g\left(x_{n(k)}\right), g\left(x_{m(k)}\right)\right), G\left(g\left(y_{n(k)}\right), g\left(y_{n(k)}\right), g\left(y_{m(k)}\right)\right)\right) \\
= & \lim _{\substack{\left.G\left(g\left(x_{n(k)}\right), g\left(x_{n(k)}\right)\right), g\left(x_{m(k)}\right)\right) \rightarrow \varepsilon_{1} \\
G\left(g\left(y_{n(k)}\right), g\left(y_{n(k)}\right), g\left(y_{m(k)}\right)\right) \rightarrow \varepsilon_{1}}} 2 \varphi\left(G\left(g\left(x_{n(k)}\right), g\left(x_{n(k)}\right), g\left(x_{m(k)}\right)\right),\right. \\
& \left.G\left(g\left(y_{n(k)}\right), g\left(y_{n(k)}\right), g\left(y_{m(k)}\right)\right)\right) \\
> & 0,
\end{aligned}
$$

which is a contradiction. Therefore, $\left\{g\left(x_{n}\right)\right\}$ and $\left\{g\left(y_{n}\right)\right\}$ are G-Cauchy sequences. By Gcompleteness of $X$, there exists $x, y \in X$ such that

$$
\lim _{n \rightarrow \infty} g\left(x_{n}\right)=x \text { and } \lim _{n \rightarrow \infty} g\left(y_{n}\right)=y \text {. }
$$

This together with the continuity of $g$ implies that

$$
\lim _{n \rightarrow \infty} g\left(g\left(x_{n}\right)\right)=g(x) \text { and } \lim _{n \rightarrow \infty} g\left(g\left(y_{n}\right)\right)=g(y)
$$

Now, suppose that assumption (a) holds. From (2.2) and the commutativity of $F$ and $g$, we obtain

$$
\begin{aligned}
g(x) & =\lim _{n \rightarrow \infty} g\left(g\left(x_{n+1}\right)\right)=\lim _{n \rightarrow \infty} g\left(F\left(x_{n}, y_{n}\right)\right)=\lim _{n \rightarrow \infty} F\left(g\left(x_{n}\right), g\left(y_{n}\right)\right) \\
& =F\left(\lim _{n \rightarrow \infty} g\left(x_{n}\right), \lim _{n \rightarrow \infty} g\left(y_{n}\right)\right) \\
& =F(x, y) .
\end{aligned}
$$

Similarly, we have

$$
\begin{aligned}
g(y) & =\lim _{n \rightarrow \infty} g\left(g\left(y_{n+1}\right)\right)=\lim _{n \rightarrow \infty} g\left(F\left(y_{n}, x_{n}\right)\right)=\lim _{n \rightarrow \infty} F\left(g\left(y_{n}\right), g\left(x_{n}\right)\right) \\
& =F\left(\lim _{n \rightarrow \infty} g\left(y_{n}\right), \lim _{n \rightarrow \infty} g\left(x_{n}\right)\right) \\
& =F(y, x) .
\end{aligned}
$$


Hence, $(x, y)$ is a coupled coincidence point of $F$ and $g$.

Finally, suppose that assumption (b) holds. Since $\left\{g\left(x_{n}\right)\right\}$ is non-decreasing satisfying $g\left(x_{n}\right) \rightarrow x$ and $\left\{g\left(y_{n}\right)\right\}$ is non-increasing satisfying $g\left(y_{n}\right) \rightarrow y$, we have

$$
g\left(g\left(x_{n}\right)\right) \preceq g(x) \text { and } \quad g(y) \preceq g\left(g\left(y_{n}\right)\right) \quad \text { for all } n \geq 0 .
$$

Using the rectangle inequality and (2.1), we get

$$
\begin{aligned}
G( & F(x, y), g(x), g(x))+G(F(y, x), g(y), g(y)) \\
\leq & G\left(F(x, y), g\left(g\left(x_{n+1}\right)\right), g\left(g\left(x_{n+1}\right)\right)\right)+G\left(g\left(g\left(x_{n+1}\right)\right), g(x), g(x)\right) \\
& +G\left(F(y, x), g\left(g\left(y_{n+1}\right)\right), g\left(g\left(y_{n+1}\right)\right)\right)+G\left(g\left(g\left(y_{n+1}\right)\right), g(y), g(y)\right) \\
= & G\left(F(x, y), g\left(F\left(x_{n}, y_{n}\right)\right), g\left(F\left(x_{n}, y_{n}\right)\right)\right)+G\left(g\left(g\left(x_{n+1}\right)\right), g(x), g(x)\right) \\
& +G\left(F(y, x), g\left(F\left(y_{n}, x_{n}\right)\right), g\left(F\left(y_{n}, x_{n}\right)\right)\right)+G\left(g\left(g\left(y_{n+1}\right)\right), g(y), g(y)\right) \\
= & G\left(F(x, y), F\left(g\left(x_{n}\right), g\left(y_{n}\right)\right), F\left(g\left(x_{n}\right), g\left(y_{n}\right)\right)\right)+G\left(g\left(g\left(x_{n+1}\right)\right), g(x), g(x)\right) \\
& G\left(F(y, x), F\left(g\left(y_{n}\right), g\left(x_{n}\right)\right), F\left(g\left(y_{n}\right), g\left(x_{n}\right)\right)\right)+G\left(g\left(g\left(y_{n+1}\right)\right), g(y), g(y)\right) \\
\leq & {\left[G\left(g(x), g\left(g\left(x_{n}\right)\right), g\left(g\left(x_{n}\right)\right)\right)+G\left(g(y), g\left(g\left(y_{n}\right)\right), g\left(g\left(y_{n}\right)\right)\right)\right] } \\
& \quad-2 \varphi\left(G\left(g(x), g\left(g\left(x_{n}\right)\right), g\left(g\left(x_{n}\right)\right)\right), G\left(g(y), g\left(g\left(y_{n}\right)\right), g\left(g\left(y_{n}\right)\right)\right)\right) \\
& +G\left(g\left(g\left(x_{n+1}\right)\right), g(x), g(x)\right)+G\left(g\left(g\left(y_{n+1}\right)\right), g(y), g(y)\right) \\
< & 0 .
\end{aligned}
$$

Letting $n \rightarrow+\infty$ in the above inequality, we obtain that

$$
G(F(x, y), g(x), g(x))+G(F(y, x), g(y), g(y))=0,
$$

which gives that $G(F(x, y), g(x), g(x))=G(F(y, x), g(y), g(y))=0$; that is, $F(x, y)=g(x)$ and $F(y, x)=g(y)$. Therefore, $(x, y)$ is a coupled coincidence point of $F$ and $g$. The proof is complete.

Setting $g(x)=x$ in Theorem 2.1, we obtain the following new result:

Theorem 2.2 Let $(X, \preceq)$ be a partially ordered set and $G$ be a G-metric on $X$ such that $(X, G)$ is a complete G-metric space. Let $F: X \times X \rightarrow X$ be a mapping having the mixed monotone property on $X$. Suppose that there exists $\varphi \in \Theta$ such that

$$
\begin{array}{r}
G(F(x, y), F(u, v), F(w, z))+G(F(y, x), F(v, u), F(z, w)) \\
\quad \leq[G(x, u, w)+G(y, v, z)]-2 \varphi(G(x, u, w), G(y, v, z))
\end{array}
$$

for all $x \succeq u \succeq w$ and $y \preceq v \preceq z$. Suppose that either

(a) $F$ is continuous or

(b) $X$ has the following property:

(i) if a non-decreasing sequence $\left\{x_{n}\right\}$ is such that $x_{n} \rightarrow x$, then $x_{n} \leq x$ for all $n$,

(ii) if a non-decreasing sequence $\left\{y_{n}\right\}$ is such that $y_{n} \rightarrow y$, then $y \preceq y_{n}$ for all $n$. 
If there exists $x_{0}, y_{0} \in X$ such that

$$
x_{0} \preceq F\left(x_{0}, y_{0}\right) \quad \text { and } \quad y_{0} \succeq F\left(y_{0}, x_{0}\right) \text {, }
$$

then F has a coupled fixed point in X.

Remark 2.3 Theorem 2.2 is more general than [37, Theorem 2.1] since the contractive condition (2.29) is weaker than (1.5), a fact which is clearly illustrated by the following example.

Example 2.4 Let $X=\mathbb{R}$ be a set endowed with order $x \preceq y \Leftrightarrow x \leq y$. Let the mapping $G: X \times X \times X \rightarrow \mathbb{R}_{+}$be defined by

$$
G(x, y, z)=|x-y|+|y-z|+|z-x|
$$

for all $x, y, z \in X$. Then $G$ is a $G$-metric on $X$. Define the mapping $F: X \times X \rightarrow X$ by

$$
F(x, y)=\frac{x-2 y}{4}, \quad \text { for all }(x, y) \in X^{2} .
$$

Then the following properties hold:

(1) $F$ is mixed monotone;

(2) $F$ satisfies condition (2.29) but $F$ does not satisfy condition (1.5).

Indeed, we first show that $F$ does not satisfy condition (1.5). Assume to the contrary, that there exists $\varphi \in \Phi$, such that (1.5) holds. This means

$$
\begin{aligned}
G(F(x, y), F(u, v), F(w, z))= & \left|\frac{x-2 y}{4}-\frac{u-2 v}{4}\right|+\left|\frac{u-2 v}{4}-\frac{w-2 z}{4}\right| \\
& +\left|\frac{w-2 z}{4}-\frac{x-2 y}{4}\right| \\
\leq & \frac{1}{2}[|x-u|+|u-w|+|w-x|+|y-v|+|v-z| \\
& +|z-y|]-\varphi((|x-u|+|u-w|+|w-x|), \\
& (|y-v|+|v-z|+|z-y|)) .
\end{aligned}
$$

Setting $x=u=w$ and $y \neq v$ or $v \neq z$ or $z \neq y$, by Remark 1.17, we get

$$
\begin{aligned}
\frac{1}{2}(|y-v|+|v-z|+|z-y|) \leq & \frac{1}{2}(|y-v|+|v-z|+|z-y|) \\
& -\varphi(0,|y-v|+|v-z|+|z-y|) \\
& <\frac{1}{2}(|y-v|+|v-z|+|z-y|),
\end{aligned}
$$

which gives a contradiction. Hence, $F$ does not satisfy condition (1.5). Now, we prove that (2.29) holds. Indeed, we have

$$
\begin{aligned}
& G(F(x, y), F(u, v), F(w, z))+G(F(y, x), F(v, u), F(z, w)) \\
& \quad=\left|\frac{x-2 y}{4}-\frac{u-2 v}{4}\right|+\left|\frac{u-2 v}{4}-\frac{w-2 z}{4}\right|+\left|\frac{w-2 z}{4}-\frac{x-2 y}{4}\right|
\end{aligned}
$$




$$
\begin{aligned}
& +\left|\frac{y-2 x}{4}-\frac{v-2 u}{4}\right|+\left|\frac{v-2 u}{4}-\frac{z-2 w}{4}\right|+\left|\frac{z-2 w}{4}-\frac{y-2 x}{4}\right| \\
\leq & \frac{1}{4}|x-u|+\frac{1}{2}|y-v|+\frac{1}{4}|u-w|+\frac{1}{2}|v-z|+\frac{1}{4}|w-x|+\frac{1}{2}|z-y| \\
& +\frac{1}{4}|y-v|+\frac{1}{2}|x-u|+\frac{1}{4}|v-z|+\frac{1}{2}|u-w|+\frac{1}{4}|z-y|+\frac{1}{2}|w-x| \\
= & \frac{3}{4}|x-u|+\frac{3}{4}|u-w|+\frac{3}{4}|w-x|+\frac{3}{4}|y-v|+\frac{3}{4}|v-z|+\frac{3}{4}|z-y| \\
= & |x-u|+|u-w|+|w-x|+|y-v|+|v-z|+|z-y| \\
& -\frac{1}{4}((|x-u|+|u-w|+|w-x|)+(|y-v|+|v-z|+|z-y|)) \\
= & {[G(x, u, w)+G(y, v, z)]-2 \varphi(G(x, u, w), G(y, v, z)) . }
\end{aligned}
$$

By the above, we get exactly (2.29) with $\varphi\left(t_{1}, t_{2}\right)=\frac{1}{8}\left(t_{1}+t_{2}\right)$.

By Theorem 2.1, we also obtain the following new result for the coupled coincidence point theorem for mixed $g$-monotone operators $F$ satisfying a contractive condition.

Theorem 2.5 Let $(X, \preceq)$ be a partially ordered set and suppose that there exists a $G$-metric $G$ on $X$ such that $(X, G)$ is a complete $G$-metric space. Let $F: X \times X \rightarrow X, g: X \rightarrow X$ so that $F$ is a mapping having the mixed $g$-monotone property on $X$. Suppose that there exists $\varphi \in \Theta$ such that

$$
\begin{aligned}
& G(F(x, y), F(u, v), F(w, z)) \\
& \leq \frac{G(g(x), g(u), g(w))+G(g(y), g(v), g(z))}{2} \\
& \quad-\varphi(G(g(x), g(u),(w)), G(g(y), g(v), g(z)))
\end{aligned}
$$

for all $g(x) \succeq g(u) \succeq g(w)$ and $g(y) \preceq g(v) \preceq g(z)$. Suppose that either

(a) $F$ is continuous or

(b) $X$ has the following property:

(i) if a non-decreasing sequence $\left\{x_{n}\right\}$ is such that $x_{n} \rightarrow x$, then $x_{n} \preceq x$ for all $n$,

(ii) if a non-decreasing sequence $\left\{y_{n}\right\}$ is such that $y_{n} \rightarrow y$, then $y \preceq y_{n}$ for all $n$.

If there exists $x_{0}, y_{0} \in X$ such that

$$
g\left(x_{0}\right) \preceq F\left(x_{0}, y_{0}\right) \text { and } g\left(y_{0}\right) \succeq F\left(y_{0}, x_{0}\right) \text {, }
$$

then $F$ and g have a coupled coincidence point.

Let $\Psi$ denote the class of all functions $\psi:[0, \infty) \rightarrow[0, \infty)$ satisfying

$$
\lim _{t \rightarrow r} \psi(t)>0 \quad \text { for all } r>0
$$

Corollary 2.6 Let $(X, \preceq)$ be a partially ordered set and $G$ be a G-metric on $X$ such that $(X, G)$ is a complete $G$-metric space. Let $g: X \rightarrow X$ be a mapping and $F: X \times X \rightarrow X$ be a mapping having the mixed $g$-monotone property on $X$. Suppose that there exists $\psi \in \Psi$ 
such that

$$
\begin{aligned}
M_{F}^{G}(x, u, w, y, v, z) \leq[ & G(g(x), g(u), g(w))+G(g(y), g(v), g(z))] \\
& -2 \psi(\max \{(G(g(x), g(u), g(w)), G(g(y), g(v), g(z)))\})
\end{aligned}
$$

for all $x, y, z, u, v, w \in X$ for which $g(x) \succeq g(u) \succeq g(w)$ and $g(y) \preceq g(v) \preceq g(z)$ where

$$
M_{F}^{G}(x, u, w, y, v, z)=G(F(x, y), F(u, v), F(w, z))+G(F(y, x), F(v, u), F(z, w)) .
$$

If there exists $x_{0}, y_{0} \in X$ such that

$$
g\left(x_{0}\right) \preceq F\left(x_{0}, y_{0}\right) \text { and } g\left(y_{0}\right) \succeq F\left(y_{0}, x_{0}\right) \text {, }
$$

and either

(a) $F$ is continuous or

(b) $X$ has the following property:

(i) if a non-decreasing sequence $\left\{x_{n}\right\}$ is such that $x_{n} \rightarrow x$, then $x_{n} \preceq x$ for all $n$,

(ii) if a non-decreasing sequence $\left\{y_{n}\right\}$ is such that $y_{n} \rightarrow y$, then $y \leq y_{n}$ for all $n$,

then $F$ and $g$ have a coupled coincidence point.

Proof In Theorem 2.1, taking $\varphi\left(t_{1}, t_{2}\right)=\psi\left(\max \left\{t_{1}, t_{2}\right\}\right)$ for all $\left(t_{1}, t_{2}\right) \in[0, \infty)^{2}$, we get the desired results.

Corollary 2.7 Let $(X, \preceq)$ be a partially ordered set and $G$ be a G-metric on $X$ such that $(X, G)$ is a complete G-metric space. Let $g: X \rightarrow X$ be a mapping and $F: X \times X \rightarrow X$ be a mapping having the mixed $g$-monotone property on $X$. Suppose that there exists $\psi \in \Psi$ such that

$$
\begin{aligned}
M_{F}^{G}(x, u, w, y, v, z) \leq & {[G(g(x), g(u), g(w))+G(g(y), g(v), g(z))] } \\
& -2 \psi(G(g(x), g(u), g(w))+G(g(y), g(v), g(z)))
\end{aligned}
$$

for all $x, y, z, u, v, w \in X$ for which $g(x) \succeq g(u) \succeq g(w)$ and $g(y) \preceq g(v) \preceq g(z)$ where

$$
M_{F}^{G}(x, u, w, y, v, z)=G(F(x, y), F(u, v), F(w, z))+G(F(y, x), F(v, u), F(z, w)) .
$$

If there exists $x_{0}, y_{0} \in X$ such that

$$
g\left(x_{0}\right) \preceq F\left(x_{0}, y_{0}\right) \quad \text { and } \quad g\left(y_{0}\right) \succeq F\left(y_{0}, x_{0}\right) \text {, }
$$

and either

(a) F is continuous or

(b) $X$ has the following property:

(i) if a non-decreasing sequence $\left\{x_{n}\right\}$ is such that $x_{n} \rightarrow x$, then $x_{n} \preceq x$ for all $n$,

(ii) if a non-decreasing sequence $\left\{y_{n}\right\}$ is such that $y_{n} \rightarrow y$, then $y \leq y_{n}$ for all $n$, then $F$ and $g$ have a coupled coincidence point.

Proof In Theorem 2.1, taking $\varphi\left(t_{1}, t_{2}\right)=\psi\left(t_{1}+t_{2}\right)$ for all $\left(t_{1}, t_{2}\right) \in[0, \infty)^{2}$, we get the desired results. 


\section{Coupled common fixed point}

Now, we shall prove the existence and uniqueness theorem of a coupled common fixed point. If $(X, \preceq)$ is a partially ordered set, we endow the product set $X \times X$ with the partial order relation:

$$
(x, y),(u, v) \in X \times X, \quad(x, y) \preceq(u, v) \quad \Leftrightarrow \quad x \preceq u, y \succeq v .
$$

Theorem 3.1 In addition to the hypotheses of Theorem 2.1, suppose that for all $(x, y)$, $\left(x^{*}, y^{*}\right) \in X \times X$, there exists $(u, v) \in X \times X$ such that $(F(u, v), F(v, u))$ is comparable with $(F(x, y), F(y, x))$ and $\left(F\left(x^{*}, y^{*}\right), F\left(y^{*}, x^{*}\right)\right)$. Then $F$ and $g$ have a unique coupled common fixed point.

Proof From Theorem 2.1, the set of coupled coincidences is non-empty. Assume that $(x, y)$ and $\left(x^{*}, y^{*}\right)$ are coupled coincidence points of $F$ and $g$. We shall show that

$$
g(x)=g\left(x^{\prime \prime}\right) \text { and } g(y)=g\left(y^{\prime \prime}\right) \text {. }
$$

By assumption, there exists $(u, v) \in X \times X$ such that $(F(u, v), F(v, u))$ is comparable with $(F(x, y), F(y, x))$ and $\left(F\left(x^{*}, y^{*}\right), F\left(y^{*}, x^{*}\right)\right)$. Putting $u_{0}=u, v_{0}=v$ and choosing $u_{1}, v_{1} \in X$ such that

$$
g\left(u_{1}\right)=F\left(u_{0}, v_{0}\right) \quad \text { and } \quad g\left(v_{1}\right)=F\left(v_{0}, u_{0}\right) .
$$

Then, similarly as in the proof of Theorem 2.1, we can inductively define sequences $\left\{g\left(u_{n}\right)\right\}$ and $\left\{g\left(v_{n}\right)\right\}$ in $X$ by

$$
g\left(u_{n+1}\right)=F\left(u_{n}, v_{n}\right) \quad \text { and } \quad g\left(v_{n+1}\right)=F\left(v_{n}, u_{n}\right), \quad \text { for all } n \geq 0 .
$$

Since $\left(F\left(x^{*}, y^{*}\right), F\left(y^{*}, x^{*}\right)\right)=\left(g\left(x^{*}\right), g\left(y^{*}\right)\right)$ and $(F(u, v), F(v, u))=\left(g\left(u_{1}\right), g\left(v_{1}\right)\right)$ are comparable, without restriction to the generality, we can assume that

$$
\left(F\left(x^{*}, y^{\prime \prime}\right), F\left(y^{\prime \prime}, x^{\prime \prime}\right)\right)=\left(g\left(x^{*}\right), g\left(y^{*}\right)\right) \preceq(F(u, v), F(v, u))=\left(g\left(u_{1}\right), g\left(v_{1}\right)\right),
$$

and

$$
(F(x, y), F(y, x))=(g(x), g(y)) \preceq(F(u, v), F(v, u))=\left(g\left(u_{1}\right), g\left(v_{1}\right)\right) .
$$

This actually means that

$$
g\left(x^{*}\right) \preceq g\left(u_{1}\right) \quad \text { and } \quad g\left(y^{*}\right) \succeq g\left(v_{1}\right)
$$

and

$$
g(x) \preceq g\left(u_{1}\right) \text { and } \quad g(y) \succeq g\left(v_{1}\right) .
$$

Using that $F$ is a mixed $g$-monotone mapping, we can inductively show that

$$
g\left(x^{*}\right) \preceq g\left(u_{n}\right) \text { and } \quad g\left(y^{*}\right) \succeq g\left(v_{n}\right) \text { for all } n \geq 1,
$$


and

$$
g(x) \preceq g\left(u_{n}\right) \quad \text { and } \quad g(y) \succeq g\left(v_{n}\right) \quad \text { for all } n \geq 1 .
$$

Thus, from (2.1), we get

$$
\begin{aligned}
G( & \left.g\left(u_{n+1}\right), g(x), g(x)\right)+G\left(g\left(v_{n+1}\right), g(y), g(y)\right) \\
= & G\left(F\left(u_{n}, v_{n}\right), F(x, y), F(x, y)\right)+G\left(F\left(v_{n}, u_{n}\right), F(y, x), F(y, x)\right) \\
= & M_{F}^{G}\left(u_{n}, x, x, v_{n}, y, y\right) \\
\leq & {\left[G\left(g\left(u_{n}\right), g(x), g(x)\right)+G\left(g\left(v_{n}\right), g(y), g(y)\right)\right] } \\
& -2 \varphi\left(G\left(g\left(u_{n}\right), g(x), g(x)\right), G\left(g\left(v_{n}\right), g(y), g(y)\right)\right),
\end{aligned}
$$

which implies that

$$
G\left(g\left(u_{n+1}\right), g(x), g(x)\right)+G\left(g\left(v_{n+1}\right), g(y), g(y)\right) \leq G\left(g\left(u_{n}\right), g(x), g(x)\right)+G\left(g\left(v_{n}\right), g(y), g(y)\right) ;
$$

that is, the sequence $\left\{G\left(g\left(u_{n}\right), g(x), g(x)\right)+G\left(g\left(v_{n}\right), g(y), g(y)\right)\right\}$ is decreasing. Therefore, there exists $\alpha \geq 0$ such that

$$
\lim _{n \rightarrow \infty}\left[G\left(g\left(u_{n}\right), g(x), g(x)\right)+G\left(g\left(v_{n}\right), g(y), g(y)\right)\right]=\alpha
$$

We shall show that $\alpha=0$. Suppose, to the contrary, that $\alpha>0$. Therefore, $\left\{G\left(g\left(u_{n}\right), g(x)\right.\right.$, $g(x))\}$ and $\left\{G\left(g\left(v_{n}\right), g(y), g(y)\right)\right\}$ have subsequences converging to $\alpha_{1}, \alpha_{2}$, respectively, with

$$
\alpha_{1}+\alpha_{2}=\alpha>0
$$

Taking the limit up to subsequences as $n \rightarrow \infty$ in (3.2), we have

$$
\alpha \leq \alpha-2 \lim _{n \rightarrow \infty} \varphi\left(G\left(g\left(u_{n}\right), g(x), g(x)\right), G\left(g\left(v_{n}\right), g(y), g(y)\right)\right)<\alpha,
$$

which is a contradiction. Thus, $\alpha=0$; that is,

$$
\lim _{n \rightarrow \infty}\left[G\left(g\left(u_{n}\right), g(x), g(x)\right)+G\left(g\left(v_{n}\right), g(y), g(y)\right)\right]=0
$$

which implies that

$$
\lim _{n \rightarrow \infty} G\left(g\left(u_{n}\right), g(x), g(x)\right)=\lim _{n \rightarrow \infty} G\left(g\left(v_{n}\right), g(y), g(y)\right)=0 .
$$

Similarly, one can show that

$$
\lim _{n \rightarrow \infty} G\left(g\left(u_{n}\right), g\left(x^{*}\right), g\left(x^{*}\right)\right)=\lim _{n \rightarrow \infty} G\left(g\left(v_{n}\right), g\left(y^{*}\right), g\left(y^{*}\right)\right)=0 .
$$

Therefore, from (3.3), (3.4) and the uniqueness of the limit, we get $g(x)=g\left(x^{*}\right)$ and $g(y)=$ $g\left(y^{*}\right)$. So, (3.1) holds. Since $g(x)=F(x, y)$ and $g(y)=F(y, x)$, by commutativity of $F$ and $g$, 
we have

$$
g(g(x))=g(F(x, y))=F(g(x), g(y)) \quad \text { and } \quad g(g(y))=g(F(y, x))=F(g(y), g(x)) .
$$

Denote $g(x)=z$ and $g(y)=w$, then by (3.5), we get

$$
g(z)=F(z, w) \quad \text { and } \quad g(w)=F(w, z) .
$$

Thus, $(z, w)$ is a coincidence point. Then from (3.1) with $x^{*}=z$ and $y^{*}=w$, we have $g(x)=$ $g(z)$ and $g(y)=g(w)$, that is,

$$
g(z)=z \quad \text { and } \quad g(w)=w .
$$

From (3.6) and (3.7), we get

$$
z=g(z)=F(z, w) \quad \text { and } \quad w=g(w)=F(w, z) .
$$

Then, $(z, w)$ is a coupled common fixed point of $F$ and $g$. To prove the uniqueness, assume that $(p, q)$ is another coupled common fixed point. Then by (3.1), we have $p=g(p)=g(z)=z$ and $q=g(q)=g(w)=w$. The proof is complete.

\section{Competing interests}

The authors declare that they have no competing interests.

\section{Authors' contributions}

All authors contributed equally and significantly in writing this paper. All authors read and approved the final manuscript.

\section{Acknowledgements}

The authors would like to thank the referees for reading this paper carefully, providing valuable suggestions and comments, and pointing out a major error in the original version of this paper. Finally, the first author is supported by the 'Centre of Excellence in Mathematics' under the Commission on Higher Education, Ministry of Education, Thailand.

\section{Received: 27 July 2012 Accepted: 17 September 2012 Published: 3 October 2012}

\section{References}

1. Banach, S: Sur les opérations dans les ensembles abstraits et leurs applications aux équations intégrales. Fundam. Math. 3, 133-181 (1922)

2. Abbas, M, Khan, $\mathrm{SH}$, Nazir, $\mathrm{T}$ : Common fixed points of $R$-weakly commuting maps in generalized metric spaces. Fixed Point Theory Appl. 2011,41 (2011)

3. Boyd, DW, Wong, JSW: On nonlinear contractions. Proc. Am. Math. Soc. 20, 458-464 (1969)

4. Mongkolkeha, C, Sintunavarat, W, Kumam, P: Fixed point theorems for contraction mappings in modular metric spaces. Fixed Point Theory Appl. 2011, 93 (2011)

5. Sintunavarat, W, Kumam, P: Weak condition for generalized multivalued $(f, \alpha, \beta)$-weak contraction mappings. Appl. Math. Lett. 24, 460-465 (2011)

6. Sintunavarat, W, Kumam, P: Gregus-type common fixed point theorems for tangential multivalued mappings of integral type in metric spaces. Int. J. Math. Math. Sci. 2011, Article ID 923458 (2011)

7. Sintunavarat, W, Kumam, P: Gregus type fixed points for a tangential multi-valued mappings satisfying contractive conditions of integral type. J. Inequal. Appl. 2011, 3 (2011)

8. Sintunavarat, W, Kumam, P: Common fixed point theorems for hybrid generalized multi-valued contraction mappings. Appl. Math. Lett. 25, 52-57 (2012)

9. Sintunavarat, W, Kumam, P: Common fixed point theorems for generalized $\mathcal{J H}$-operator classes and invariant approximations. J. Inequal. Appl. 2011, 67 (2011)

10. Dukić, D, Kadelburg, Z, Radenović, S: Fixed point of Geraghty-type mappings in various generalized metric spaces. Abstr. Appl. Anal. 2011, Article ID 561245 (2011). doi:10.1155/2011/561245

11. Ran, ACM, Reurings, MCB: A fixed point theorem in partially ordered sets and some applications to matrix equations. Proc. Am. Math. Soc. 132, 1435-1443 (2004)

12. Nieto, JJ, Lopez, RR: Existence and uniqueness of fixed point in partially ordered sets and applications to ordinary differential equations. Acta Math. Sin. Engl. Ser. 23(12), 2205-2212 (2007) 
13. Agarwal, RP, El-Gebeily, MA, O’Regan, D: Generalized contractions in partially ordered metric spaces. Appl. Anal. 87, $1-8(2008)$

14. Altun, I, Simsek, H: Some fixed point theorems on ordered metric spaces and application. Fixed Point Theory Appl. 2010, Article ID 621469 (2010)

15. Harjani, J, Sadarangani, K: Generalized contractions in partially ordered metric spaces and applications to ordinary differential equations. Nonlinear Anal. 72, 1188-1197 (2010)

16. Kadelburg, Z, Pavlović, M, Radenović, S: Common fixed point theorems for ordered contractions and quasicontractions in ordered cone metric spaces. Comput. Math. Appl. 59, 3148-3159 (2010)

17. Radenović, S, Kadelburg, Z: Generalized weak contractions in partially ordered metric spaces. Comput. Math. Appl. 60, 1776-1783 (2010)

18. Sintunavarat, W, Cho, YJ, Kumam, P: Common fixed point theorems for c-distance in ordered cone metric spaces. Comput. Math. Appl. 62, 1969-1978 (2011)

19. Sintunavarat, W, Cho, YJ, Kumam, P: Coupled fixed point theorems for weak contraction mappings under F-invariant set. Abstr. Appl. Anal. 2012, Article ID 324874 (2012)

20. Berinde, $\mathrm{V}$ : Coupled coincidence point theorems for mixed monotone nonlinear operators. Comput. Math. Appl. (2012). doi:10.1016/j.camwa.2012.02.012

21. Bhaskar, TG, Lakshmikantham, V: Fixed point theorems in partially ordered metric spaces and applications. Nonlinear Anal. 65, 1379-1393 (2006)

22. Abbas, M, Khan, AR, Nazir, T: Coupled common fixed point results in two generalized metric spaces. Appl. Math. Comput. 217, 6328-6336 (2011)

23. Nashine, HK, Kadelburg, Z, Radenović, S: Coupled common fixed point theorems for $w^{*}$-compatible mappings in ordered cone metric spaces. Appl. Math. Comput. 218, 5422-5432 (2012)

24. Shatanawi, W, Abbas, M, Nazir, T: Common coupled fixed points results in two generalized metric spaces. Fixed Point Theory Appl. 2011, 80 (2011). doi:10.1186/1687-1812-2011-80

25. Sintunavarat, W, Cho, YJ, Kumam, P: Coupled coincidence point theorems for contractions without commutative condition in intuitionistic fuzzy normed spaces. Fixed Point Theory Appl. 2011, 81 (2011)

26. Mustafa, Z, Sims, B: Some remarks concerning D-metric spaces. In: Proc. Int. Conf. on Fixed Point Theor. Appl., Valencia, Spain 189-198 (2003)

27. Mustafa, Z, Sims, B: A new approach to generalized metric spaces. J. Nonlinear Convex Anal. 7(2), 289-297 (2006)

28. Abbas, M, Rhoades, BE: Common fixed point results for noncommuting mappings without continuity in generalised metric spaces. Appl. Math. Comput. 215, 262-269 (2009)

29. Abbas, M, Nazir, T, Radenovic, S: Some periodic point results in generalized metric spaces. Appl. Math. Comput. 217(8), 4094-4099 (2010)

30. Chugh, R, Kadian, T, Rani, A, Rhoades, BE: Property P in G-metric spaces. Fixed Point Theory Appl. 2010, Article ID 401684 (2010)

31. Mustafa, Z, Shatanawi, W, Bataineh, M: Fixed point theorems on uncomplete G-metric spaces. J. Math. Stat. 4(4), 196-201 (2008)

32. Mustafa, Z, Shatanawi, W, Bataineh, M: Existence of fixed point result in G-metric spaces. Int. J. Math. Math. Sci. 2009, Article ID 283028 (2009)

33. Mustafa, Z, Sims, B: Fixed point theorems for contractive mappings in complete G-metric space. Fixed Point Theory Appl. 2009, Article ID 917175 (2009)

34. Saadati, R, Vaezpour, SM, Vetro, P, Rhoades, BE: Fixed point theorems in generalized partially ordered G-metric spaces. Math. Comput. Model. 52, 797-801 (2010)

35. Lakshmikantham, V, Ćirić, LJ: Coupled fixed point theorems for nonlinear contractions in partially ordered metric spaces. Nonlinear Anal. 70, 4341-4349 (2009)

36. Choudhury, BS, Maity, P: Coupled fixed point results in generalized metric spaces. Math. Comput. Model. 54, 73-79 (2011)

37. Luong, NV, Thuan, NX: Coupled fixed point theorems in partially ordered G-metric spaces. Math. Comput. Model. 55, 1601-1609 (2012)

doi:10.1186/1687-1812-2012-172

Cite this article as: Wangkeeree and Bantaojai: Coupled fixed point theorems for generalized contractive mappings in partially ordered G-metric spaces. Fixed Point Theory and Applications 2012 2012:172.

\section{Submit your manuscript to a SpringerOpen ${ }^{\odot}$ journal and benefit from:}

- Convenient online submission

Rigorous peer review

Immediate publication on acceptance

- Open access: articles freely available online

- High visibility within the field

- Retaining the copyright to your article

Submit your next manuscript at $>$ springeropen.com 